\title{
OVARIAN STIMULATION AND ULTRASOUND-GUIDED OOCYTE RETRIEVAL IN BABOON (PAPIO CYNOCEPHALUS ANUBIS) DURING PITUITARY SUPPRESSION WITH A GnRH AGONIST ${ }^{*}$
}

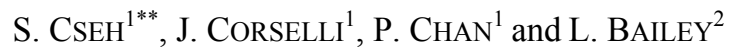 \\ ${ }^{1}$ Department of Obstetrics and Gynaecology, Faculty of Veterinary Science Budapest, \\ Szent István University, H-1400 Budapest, P.O. Box 2, Hungary; ${ }^{2}$ Department \\ of Surgery, School of Medicine, Loma Linda University, Loma Linda, CA, USA, 92350
}

(Received April 18, 2001; accepted September 5, 2001)

\begin{abstract}
The objective of this study was to investigate whether baboon females respond to an ovarian stimulation protocol incorporating pituitary suppression with a GnRH agonist (GnRHa) and either highly purified human FSH (hphFSH) or recombinant human FSH (rhFSH) with follicular development and oocyte maturation. A modified human ovulation induction protocol was applied to 5 adult female baboons with a history of regular menstrual cycles (33-34 days). A long-acting GnRHa implant containing goserelin acetate was placed subcutaneously (s.c.) on Days 22-24 of their menstrual cycle. Concentrations of serum oestradiol (E2), progesterone (P4) and human FSH were obtained by ELISA. Menses occurred $\sim 10$ days after GnRHa implantation. Daily hphFSH or rhFSH (75 IU i.m.) treatments were started $\sim 10$ days following menses. When the majority of follicles were $\geq 5 \mathrm{~mm}$ in diameter and the E2 levels had reached a maximum, hCG (2000 IU i.m.) was administered to induce final maturation of oocytes and ovulation. Thirty to $34 \mathrm{~h}$ after hCG administration, transabdominal follicular aspiration was performed using a variable frequency transvaginal transducer with ultrasound. A total of 71 oocytes were collected from 4 animals (average: 17). The meiotic maturity of oocytes was evaluated $3 \mathrm{~h}$ after retrieval. Ninety-one percent of oocytes were in metaphase 2 and of grades I and II which are appropriate for in vitro insemination.
\end{abstract}

Key words: Ovarian stimulation, GnRH agonist, ultrasound-guided oocyte retrieval, baboon

New protocols for superovulation and oocyte production have been developed over the last decade for assisted reproduction. One of the major advances has been the incorporation of gonadotropin releasing hormone agonists (GnRHa) to suppress pituitary control of ovarian function. The continued administration of $\mathrm{GnRH}$ as goserelin acetate, buserelin acetate or leuprolide acetate causes an initial

\footnotetext{
*The data were partly presented at the annual meeting of IETS in Maastricht, in 2000

${ }^{* *}$ Corresponding author; Dr. Sándor Cseh, H-1400 Budapest, Pf. 2, Hungary; E-mail: scseh@univet.hu; Fax: +36 (26) 360-499
} 
surge of gonadotropin release followed by long-term suppression of gonadotropin release as GnRH receptors are internalised (Meldrum, 1991). These procedures have been developed for human use and limited data are available to show the efficacy of these treatments in nonhuman primates.

In farm animals, GnRH or its analogues are used successfully in several fields of reproduction, such as oestrus synchronisation, treatment of cystic ovarian disease and stimulating follicular development, etc. The advantages of the use of GnRH in contrast to LH or hCG are: small molecular size, it is not likely stimulate an immune response, therefore repeated treatment with GnRH or its analogues does not result in refractoriness and is less expensive than that of LH or hCG (Brüssow et al., 1994; Youngquist, 1997).

Human and nonhuman primate (NHP) oocytes are generally retrieved by follicular aspiration before ovulation following controlled ovarian stimulation (COS) for assisted reproduction. Follicular aspiration in NHP has been performed by laparotomy or laparoscopy (Bavister et al., 1983; Bavister and Boatman, 1989; Clayton and Kuehl, 1984; Lopata et al., 1988; VandeVoort et al., 1989; Wolf et al., 1989; Wolf et al., 1990). Recently, successful transabdominal ultrasound-guided follicular aspiration has been reported in macaque (VandeVoort and Tarantal, 1991). However, no ultrasound-guided follicular aspiration has been published in baboon yet.

NHP gonadotropin preparations are not available, thus foreign gonadotropins (e.g. PMSG or human), usually human, are generally used to induce ovarian stimulation (Dukelow and Vengesa, 1986; Wolf et al., 1990). Human menopausal gonadotropin (menotropin, Pergonal ${ }^{\circledR}$, Serono, Randolph, MA), a urinary extract containing equivalent amounts of FSH and LH activity, and human urofollitropin (Metrodin ${ }^{\circledR}$, Serono, 1995) containing primarily FSH have been used for baboon COS (Fourie et al., 1987; McCarthy et al., 1991). No data are available on COS with GnRHa and hphFSH or rhFSH in baboon.

The objectives of this study were (1) to investigate whether baboon females respond to an ovarian stimulation protocol incorporating pituitary suppression with a GnRH agonist and either highly purified human FSH (hphFSH) or recombinant human FSH (rhFSH) with follicular development and oocyte maturation, and (2) to evaluate the effectiveness of using a transvaginal transducer for transabdominal ultrasound-guided oocyte retrieval in baboons.

\section{Materials and methods}

\section{Controlled Ovarian Stimulation}

A modified human ovulation induction protocol was applied to five adult female baboons ( 6 to 15 years of age, 12 to $17 \mathrm{~kg}$ ) with a history of regular menstrual cycles (32 to 34 days) (Fig. 1). The animals were caged alone in a con- 
trolled environment $\left(25-27{ }^{\circ} \mathrm{C}\right.$, humidity $70 \%, 12 / 12$ hour light/dark cycle). Their menstrual cycle was monitored by observation of perineal sex skin turgeses (follicular phase) and deturgeses (after ovulation). Cycle day 1 (CD 1) was the first day menses was observed. A long-acting GnRHa implant containing goserelin acetate $\left(3.6 \mathrm{mg}\right.$, Zoladex ${ }^{\circledR}$, ICI Pharma, Wilmington, DE) was placed s.c. on CD 22-24 (luteal phase) of their menstrual cycle. Menses occurred about 10 days after GnRHa implantation. Following menses, on Days 3 and 6, the serum E2 levels were checked. Daily hphFSH (Fertinex ${ }^{\mathrm{TM}}$, Serono; 2 animals, 75 IU, i.m.) or rhFSH ( 3 animals, 75 IU, i.m.) were started $\sim 10$ days following menses. When the majority of follicles were $>5 \mathrm{~mm}$ in diameter and the E2 levels reached their maximum, $2000 \mathrm{IU}$ hCG (Profasi ${ }^{\circledR}$, Serono) was administered i.m. to induce final oocyte maturation and ovulation.

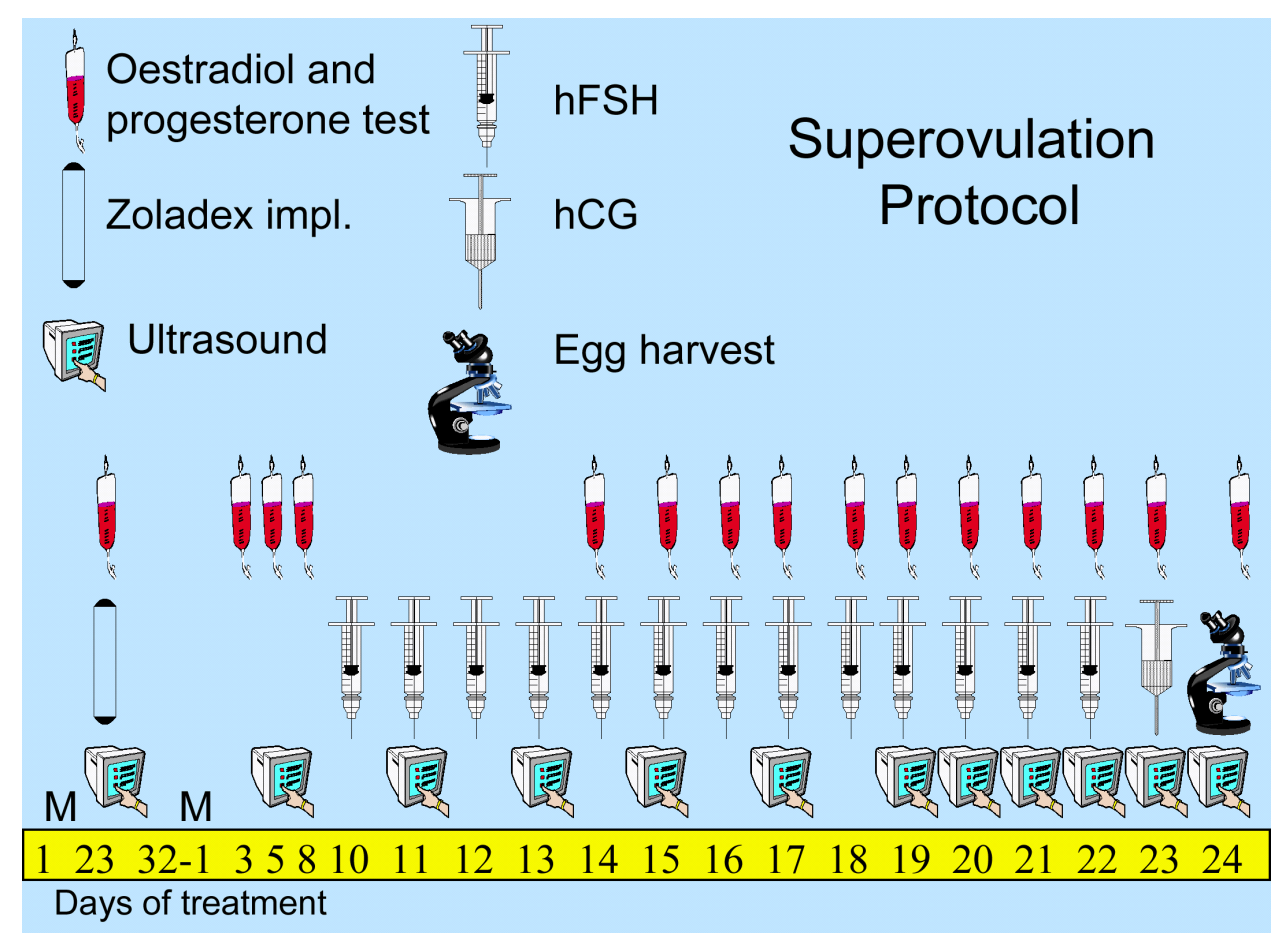

Fig. 1. Superovulation protocol

\section{Serum hormone test}

Non-isotopic methods were used to measure serum levels of oestradiol (E2), progesterone (P4) and hFSH. Serum E2, P4 and hFSH levels were obtained by immunoassay (ELISA) (DPC, Diagnostic Products Corporation, Los Angeles, USA). The inter-assay coefficient of variation over the calculated range of the assay did not exceed $8 \%$. 


\section{Egg retrieval}

Transabdominal follicular aspiration was performed 30 to $34 \mathrm{~h}$ after hCG administration using a variable frequency transvaginal transducer (Endo-V transducer, 5.0/6.0/7.5 MHZ; Siemens, Isaaquah, WA) with an ultrasound system (Siemens Sonoline SI-250). The follicular contents were aspirated into culture tubes using a disposable 17-gauge $30 \mathrm{~cm}$ long double-lumen needle connected to tubing (Swemedlab, Frounda, Sweden) and a suction pump (Rocket, Alpharetta, GA) set at $100 \mathrm{~mm} \mathrm{Hg}$. Follicles were rinsed with modified human tubal fluid (mHTF, Irvine Scientific, Irvine, CA) at $37{ }^{\circ} \mathrm{C}$ supplemented with $10 \%$ synthetic serum substitute (SSS, Irvine Scientific, Irvine, CA). Oocytes were assessed for meiotic maturity $3 \mathrm{~h}$ after retrieval. Oocytes were examined for presence of cytoplasmic vesicles and evidence of nuclear maturation. Only oocytes that had extruded a first polar body were considered appropriate for in vitro insemination.

\section{Results}

Serum levels of P4 in all cases of COS were between 10.3 and $13.8 \mathrm{ng} / \mathrm{mL}$ at the beginning of the GnRHa treatments indicating that the animals were in the luteal phase which was suitable to start pituitary suppression. GnRHa pituitary suppression was evident by low E2 levels $(<20 \mathrm{pg} / \mathrm{mL})$ and absence of perineal turgeses in the days just prior to FSH injection. Figure 2 shows the E2 concentrations in five baboon females during the COS cycles. The serum E2 levels in all COS cycles, except one, increased 4 to 17 -fold to a peak around Days 9 and 12 post gonadotropin administration. E2 levels began to rise 5 to 7 days after beginning FSH treatment. Sonographic evidence of the follicular development was not observed transvaginally or transabdominally until treatment Days 9 to 10 when several follicles 2 to $3 \mathrm{~mm}$ in diameter and multiple smaller follicles became visible. The serum E2 reached plateau levels of 204, 207, 306 and $966 \mathrm{pg} / \mathrm{mL}$ between treatment Days 8 to 12. Follicle sizes increased to $>5 \mathrm{~mm}$ between treatment Days 9 and 12 and hCG was administered (Fig. 3).

Figure 4 shows the circulating human FSH concentrations detected in serum of four baboon females during COS beginning at the time of the first intramuscular injection of either $75 \mathrm{IU}$ hphFSH or rhFSH. The values increased very rapidly during the first treatment day, then moderately rapidly. The serum hFSH reached plateau levels of 11 to 13 mIU between treatment Days 9 to 11 . 


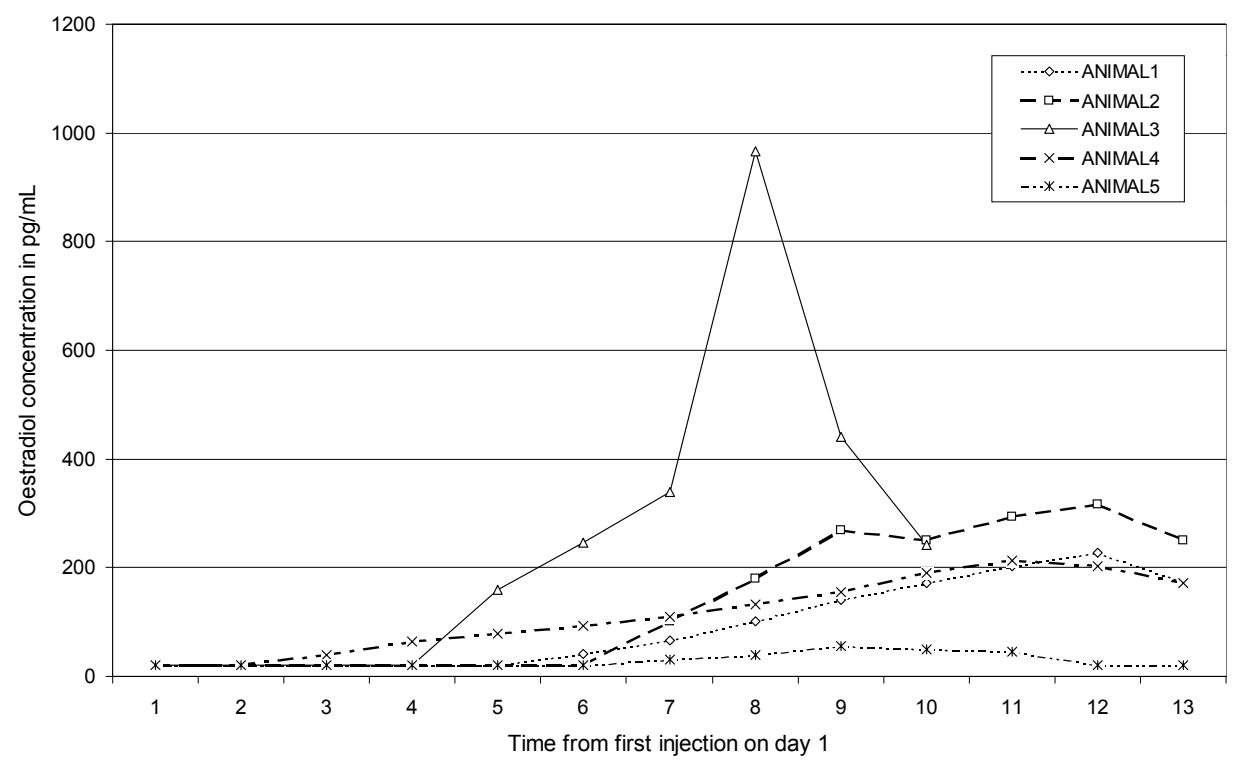

Fig. 2. Serum oestradiol concentrations in the five baboon females during controlled ovarian stimulation

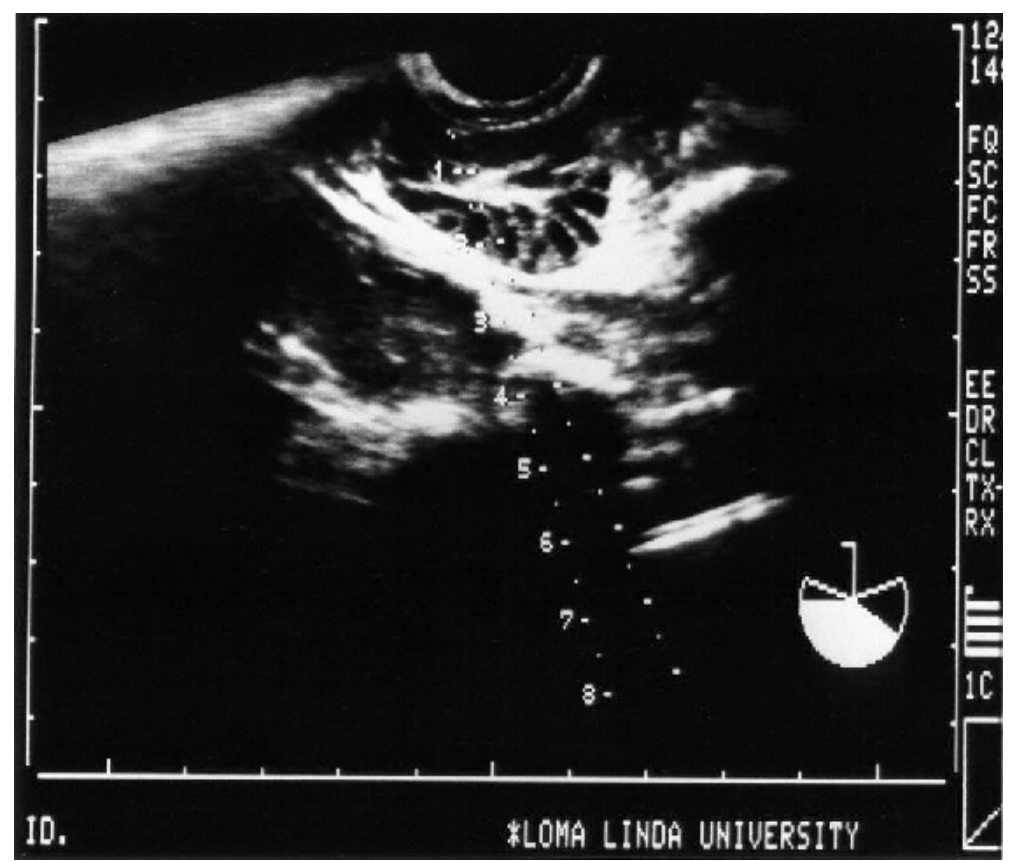

Fig. 3. Representative ultrasonogram of a baboon ovary containing multiple follicles, just before aspiration 


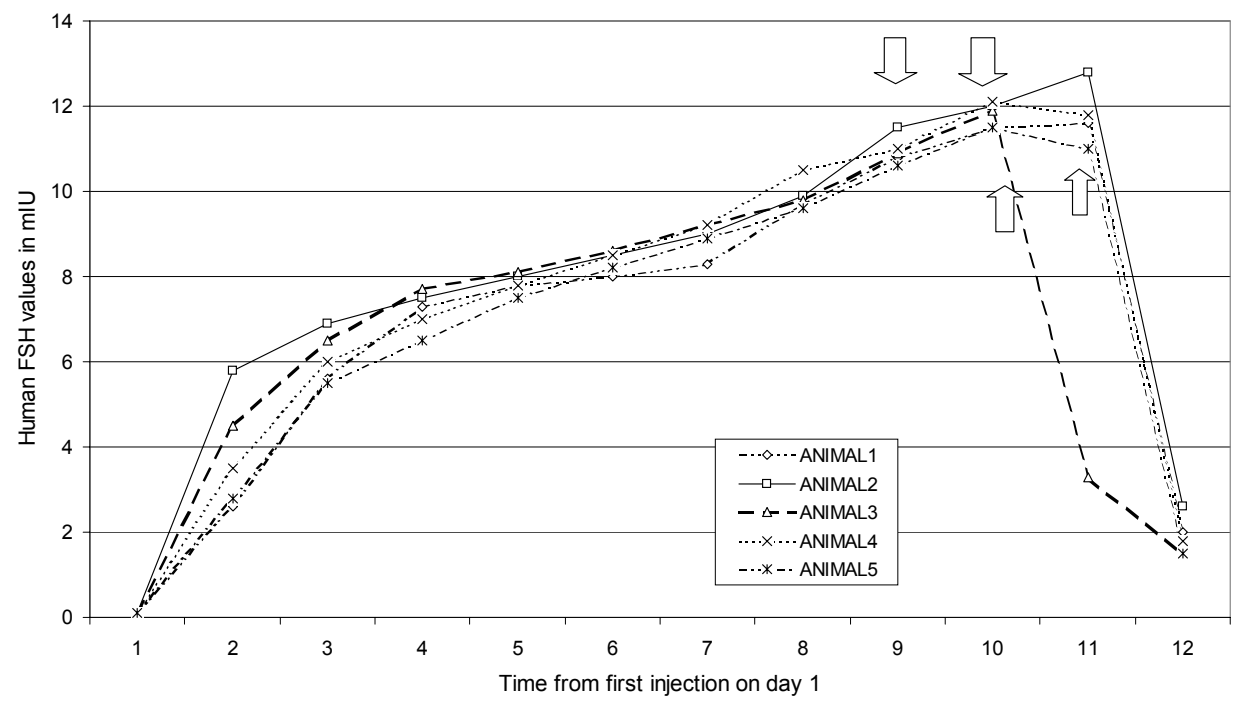

Fig. 4. Human FSH concentrations detected in serum during controlled ovarian stimulation of five baboon females

Follicular development was followed with ultrasonography. Follicular aspiration was carried out from 4 of five treated animals between 30 to $34 \mathrm{~h}$ after hCG injection. One animal did not respond to the treatment and the ovarian stimulation was cancelled. Fourteen, 21,16 and 20 oocytes $(n=71)$ were collected from 4 animals. The average recovery rate of oocytes from the rinsed follicles was $86 \%$ (71/82). Ninety-one percent of the collected oocytes were morphologically normal, all in metaphase 2 and of grades I and II, which are considered appropriate for in vitro insemination. An average of 17 ova was obtained from each treatment cycle.

\section{Discussion}

This study was undertaken to test the hypothesis that baboon females will respond with follicular development and oocyte maturation to a modified human controlled ovarian stimulation protocol incorporating a luteal phase start with a GnRHa followed by injection of either hphFSH or rhFSH. Our results demonstrate that a $3.6 \mathrm{mg}$ Zoladex ${ }^{\circledR}$ implant provided sufficient GnRHa to suppress pituitary control of ovarian function in baboon females. As in the human female, initiation of GnRHa in the luteal phase prevents follicular development and E2 secretion following menses which was documented by the low serum level of E2 and the suppressed baboon perineal sex skin turgescence. Our data indicate that during GnRHa suppression, baboon females are able to respond to the human gonadotropin treatment. The circulating serum E2 levels increased 4 to 17-fold to 
a peak and this elevation was sufficient to stimulate sex skin turgescence. The data indicate that highly purified human FSH and recombinant human FSH induce follicular development associated with E2 production in the baboon ovary. Our results show that 9 to 10 days injection of either $75 \mathrm{IU}$ hphFSH or rhFSH per day produced peak E2. Daily injections of FSH resulted in a plateau of serum values by 8 to 9 days of treatment. A similar pattern has been observed in women treated with daily injections of hFSH (Porchet et al., 1994).

In this preliminary study, ova were used for a variety of pilot experiments such as intracytoplasmic sperm injection (ICSI). Using ICSI, we could demonstrate that the collected oocytes are capable of fertilisation. This indicates that sufficient exposure to in vivo conditions was provided and the quality of ova obtained appropriate for in vitro fertilisation.

In summary, highly purified human FSH or recombinant human FSH effectively induce COS and oocyte development in the baboon suppressed with GnRHa. The variations in hormone profiles during ovarian stimulation were similar to those found in humans, but the E2 levels measured were lower. The depot form of GnRHa is effective and beneficial for baboons because the requirement of daily animal access for administration by injection is minimised. Transabdominal ultrasound-guided follicular aspiration using a transvaginal transducer is effective and safe for oocyte retrieval in baboon. Morphologically normal mature oocytes in metaphase 2 and of grades I and II can be obtained and they are capable of fertilisation.

\section{Acknowledgements}

The authors are grateful to Thomas J. Kuehl Ph.D. (A \& M University, College of Medicine) for providing helpful discussion during this project. We are also grateful to Serono Laboratories for providing the recombinant human FSH to the experiments. We are very thankful to Katy Brasky DVM (Southwest Foundation for Biomedical Research) for her help. We wish to thank Geoffrey Visbal, Nancy Holmqvist, Joyce Ivy and Jan Smallwood for excellent technical assistance. We also wish to thank Evelyn Agcaoli, Lane Beha and Simran Bal Toor for conducting the ELISA.

\section{References}

Bavister, B. D. and Boatman, D. E. (1989): In vitro fertilization and embryo transfer technology as an aid to the conservation of endangered primates. Zoo Biol. (Suppl.), 21-31.

Bavister, B. D., Boatman, D. E., Leibfried, L., Loose, M. and Vernon, M. W. (1983): Fertilization and cleavage of rhesus monkey oocytes in vitro. Biol. Reprod. 28, 983-999.

Brüssow, K., Schneider, F. and Rátky, J. (1994): The influence of longtime active GnRH-agonist on hormonal level and follicular development in cyclic gilts. Reprod. Dom. Anim. 29, 358-361. 
Clayton, O. and Kuehl, T. J. (1984): The first successful in vitro fertilization and embryo transfer in a nonhuman primate. Theriogenology 21, 228 (abstract).

Dukelow, W. R. and Vengesa, P. N. (1986): Primate models for fertilization and early embryogenesis. In: Benirschke, K. (ed.) Primates: The road to self-sustaining populations. Springer-Verlag, New York, pp. 445-461.

Fertinex $^{\text {TM }}$ (urofollitropin for injection, purified) Package insert, 1996. Serono Laboratories, Inc. Randolph, MA 02368 USA.

Fourie, F. R., Snyman, E., Van Der Merwe, J. V. and Grace, A. (1987): Primate in vitro fertilization research: Preliminary results on the folliculogenic effects of three different ovulatory induction agents on the chacma baboon, Papio ursinus. Comp. Biochem. Physiol. 87A, 889-893.

Lopata, A., Summers, P. M. and Hearn, J. P. (1988): Births following the transfer of cultured embryos obtained by in vitro and in vivo fertilization in the marmoset monkey (Callithrix jacchus). Fertil. Steril. 50, 503-509.

McCarthy, T. J., Fortman, J. D., Boice, M. L., Fazlebas, A. T. and Verhage, H. G. (1991): Induction of multiple follicular development and superovulation in the olive baboon, Papio anubis. J. Med. Primatol. 20, 308-314.

Meldrum, D. (1991): Use of GnRH agonists in programs of ovulation induction and controlled ovarian hyperstimulation. In: Barbieni, R. L. and Friedman, A. J. (eds) Gonadotropin Releasing Hormone Analogous Applications in Gynecology. Elsevier Science Publishing Co., New York, pp. 91-102.

Metrodin $^{\circledR}$ (urofollitropin for injection) Package insert, 1995. Serono Laboratories, Inc. Randolph, MA 02368 USA.

Porchet, H. C., Cotonnec, J. V. and Loumaye, E. (1994): Clinical pharmacology of recombinant human follicle stimulating hormone. III. Pharmacokinetic-pharmacodynamic modeling after repeated subcutaneous administration. Fertil. Steril. 61, 687-695.

Youngquist, R. S. (1997): Current Therapy in Large Animal Theriogenology. W. B. Saunders Company, Philadelphia, USA.

VandeVoort, C. A. and Tarantal, A. F. (1991): The macaque model for in vitro fertilization: Superovulation techniques and ultrasound-guided follicular aspiration. J. Med. Primatol. 20, $110-116$.

VandeVoort, C. A., Baugham, W. L. and Stoufer, R. L. (1989): Comparison of different regimens of human gonadotropins for superovulation of rhesus monkeys: ovulatory response and subsequent luteal function. J. In Vitro Fertil. Embryo Transfer 6, 85-91.

Wolf, D. P., Thomson, J. A., Zelinski-Wooten, M. B. and Stoufer, R. L. (1990): In vitro fertilization - embryo transfer in nonhuman primates: the technique and its application. Mol. Reprod. Dev. 27, 261-280.

Wolf, D. P., VandeVoort, C. A., Meyer-Haas, G. R., Zelinski-Wooten, M. B., Hess, D. L., Baugham, W. L. and Stoufer, R. L. (1989): In vitro fertilization and embryo transfer in the rhesus monkey. Biol. Reprod. 41, 335-346. 